\title{
Genomic regulation of natural variation in cortical and noncortical brain volume
}

\author{
Jackson Beatty* and Rick E Laughlin
}

Address: Department of Psychology, University of California Los Angeles, Los Angeles, California, USA

Email: Jackson Beatty* - beatty@ucla.edu; Rick E Laughlin - rlaugh@ucla.edu

* Corresponding author

Published: 17 February 2006

BMC Neuroscience 2006, 7:16 doi:10.1186/1471-2202-7-16

This article is available from: http://www.biomedcentral.com/I47/-2202/7//6

(c) 2006 Beatty and Laughlin; licensee BioMed Central Ltd.

This is an Open Access article distributed under the terms of the Creative Commons Attribution License (http://creativecommons.org/licenses/by/2.0), which permits unrestricted use, distribution, and reproduction in any medium, provided the original work is properly cited.
Received: 08 September 2005

Accepted: 17 February 2006

\begin{abstract}
Background: The relative growth of the neocortex parallels the emergence of complex cognitive functions across species. To determine the regions of the mammalian genome responsible for natural variations in cortical volume, we conducted a complex trait analysis using 34 strains of recombinant inbred (RI) strains of mice (BXD), as well as their two parental strains (C57BL/6) and DBA/2J). We measured both neocortical volume and total brain volume in 155 coronally sectioned mouse brains that were Nissl stained and embedded in celloidin. After correction for shrinkage, the measured cortical and noncortical brain volumes were entered into a multiple regression analysis, which removed the effects of body size and age from the measurements. Marker regression and interval mapping were computed using WebQTL.
\end{abstract}

Results: An ANOVA revealed that more than half of the variance of these regressed phenotypes is genetically determined. We then identified the regions of the genome regulating this heritability. We located genomic regions in which a linkage disequilibrium was present using WebQTL as both a mapping engine and genomic database. For neocortex, we found a genome-wide significant quantitative trait locus (QTL) on chromosome I I (marker DI IMit l9), as well as a suggestive QTL on chromosome 16 (marker DI6Mit I00). In contrast, for noncortex the effect of chromosome II was markedly reduced, and a significant QTL appeared on chromosome 19 (DI 9Mit22).

Conclusion: This classic pattern of double dissociation argues strongly for different genetic factors regulating relative cortical size, as opposed to brain volume more generally. It is likely, however, that the effects of proximal chromosome II extend beyond the neocortex strictly defined. An analysis of single nucleotide polymorphisms in these regions indicated that ciliary neurotrophic factor ( $(n t f)$ is quite possibly the gene underlying the noncortical QTL. Evidence for a candidate gene modulating neocortical volume was much weaker, but Otxl deserves further consideration.

\section{Background}

\section{Cortex and cognition}

The absolute and relative volumes of anatomically defined brain regions-such as the mammalian cerebral cortex-are of functional importance both within and across species [1-4]. In humans, the volume of the cere- bral hemispheres ranges between 850 and $1380 \mathrm{~cm}^{3}$ in young adults [5]. Further, neocortical size is hereditarily specified, with over eighty percent of the variance of human neocortical gray matter volume being genetically determined [6]. At present, little is known about the genomic determinants of such natural variation. 
Further, cognitive ability is related to neocortical size. For example, Reiss et al. showed that IQ is positively correlated with cerebral volume in children [7]. Thompson et al. provided evidence based on quantitative MRI volumetric measurements that not only is neocortical volume genetically determined $\left(h^{2}>0.8\right)$, but that Spearman's $g$, a measure of fluid intelligence, was significantly linked to frontal lobe neocortical volume. Similar findings also have been reported by Posthuma et al. [9].

These results provide evidence that neocortical volume is very much genetically determined and linked to cognitive abilities. However, such studies provide no evidence concerning the genomic mechanisms that underlie these highly heritable traits. For this all important question, quantitative neuroanatomical studies of the neocortex of recombinant inbred strains of mice provide one important path to unravelling the genomics of brain size. The discovery of the genes that differentially regulate neocortical volume is a primary question for contemporary cognitive neuroscience. The study of RI mice might provide some insight into this problem. Here, we report the first empirical study of this fundamental problem.

\section{Experimental strategy}

We measured neocortex and total brain volume in 155 mice from $34 \mathrm{RI}$ strains (BXD) as well as their two parental strains, C57BL/6J (B) and DBA/2J (D), all strains being homozygous throughout their genomes. From these measurements, both in vivo cortical brain and in vivo noncortical brain volumes were calculated.

\section{Results}

\section{Reliability of measurement}

To assess the reliability of the stereological measurements, cortical brain area was remeasured blindly in 94 brain sections. The test-retest reliability coefficient indicated that the measurements were highly reliable $(r=0.984)$. Similarly, reliability for total fixed brain volume remeasured for twenty mice was also very high $(r=0.996)$.

\section{Regression analyses}

The size of brain structure is not only regulated by structure-specific genes, but varies with other factors, which may include body weight (BW), age, and sex. To statistically remove these influences from our histological phenotypes, a multiple-regression analysis was performed using body weight, the logarithm of age, and sex as predictor variables, a standard procedure in QTL analysis. Body weight and the logarithm of age were the only significant predictor variables for in vivo cortical brain volume, yielding the following regression equation for adjusted cortical volume:

$C_{x} V_{\text {Reg }}=111.68+1.16(B W)-18.38(\ln (A g e))$ henceforth referred to as neocortex.

The same two predictor variables were used for the regression of in vivo noncortical brain volume, although body weight was the only significant predictor in that analysis. The regression equation for in vivo noncortical brain volume was:

$N C_{x} V_{\text {Reg }}=270.53+3.87(B W)-17.86(\ln ($ Age $))$,

termed noncortex in the following analyses.

\section{QTL mapping}

The linkage disequilibrium between a measured phenotypic trait, like regressed cortical volume or regressed noncortical volume, and any genomic marker can be measured using the likelihood ratio statistic (LRS). This is a quantitative estimate of the probability that each individual marker does or does not overlie one of the polygenes regulating the phenotypic trait.

We computed the LRS for each of 3,795 marker positions on chromosomes 1 through 19 as well as chromosome X, using webQTL as a genotype source and mapping engine $[10,11]$. Similar results were also obtain in preliminary analyses using the previous marker set containing 788 more sparsely distributed WebQTL markers.

The LRS depends upon the ratio of a model not incorporating information about the marker in question (the reduced model) to a model incorporating genetic information (the full model), as shown in Equation 3. In marker regression, the LRS for a particular marker is a function of the ratio of the sum of squared deviations from the mean for the phenotypic variable $x$ to the sum of the squared deviations of the variable from the submeans that are obtained when the phenotypes are segregated according to the parental allele value at that marker:

$$
\begin{aligned}
L R S & =N \ln \frac{S S_{\text {ReducedModel }}}{S S_{\text {FullModel }}} \\
& =N \ln \frac{\sum\left(x_{A}-\bar{x}_{A}\right)^{2}}{\sum\left(x_{B}-\bar{x}_{B}\right)^{2}+\sum\left(x_{D}-\bar{x}_{D}\right)^{2}}
\end{aligned}
$$

where the subscripts $A, B$, and D indicate, respectively, all strains, strains with the C57BL/6J B-type, and strains with the DBA/2J D-type allele at the marker, as shown above. $\mathrm{N}$ is the total number of strains analyzed, here 36 . The value of the LRS can be compared with values obtained from a permutation test to determine the genome-wide significance of the association [12]. 
Table 2: Effects of Strain on Cortical and Noncortical Volume. The effects of strains (genome) on both cortex and noncortex were analysed by analysis of variance. Both effects were highly significant.

\begin{tabular}{lrrrrr}
\hline Source & Sum of Squares & df & Mean Square & F & Significance \\
\hline Strain & 12287.62 & 35 & 351.07 & 5.91 & .0001 \\
Error & 7069.61 & 119 & 59.41 & & \\
Total & 19357.23 & 154 & & & \\
\hline
\end{tabular}

Residual noncortical brain volume:

\begin{tabular}{lrrrrr}
\hline Source & Sum of Squares & df & Mean Square & F & Significance \\
\hline Strain & 73934.94 & 35 & 2112.43 & 8.73 & .0001 \\
Error & 28806.79 & 119 & 242.07 & & \\
Total & 102741.73 & 154 & & & \\
\hline
\end{tabular}

\section{QTL boundaries}

The boundaries of each QTL were determined by examination of the distribution of the LRS scores of the markers surrounding the QTL peak. All peaks were well formed and sharp. Following Van Ooijen [13] we located the nearest markers for which the value of the LRS was reduced by at least 9.2 units, corresponding to the 95 percent confidence limits for the QTL. Kim et al. has recently confirmed that this method is both accurate and unbiased [14].

\section{Descriptive statistics}

There was substantial variation in both in vivo neocortical and noncortical brain volume across strains. In vivo neocortical volume ranged from a minimum of $77.3 \mathrm{~mm}^{3}$ in BXD30 mice to $125.1 \mathrm{~mm}^{3}$ in BXD5 mice.

The mean in vivo neocortical volume was $101.9 \mathrm{~mm}^{3}$, with a standard deviation of 9.8. In vivo noncortical brain volume ranged from 266.6 (BXD27) to $391.7 \mathrm{~mm}^{3}$ (BXD5), with a mean of 319.9 and a standard deviation of 25.2. The range of both phenotypic measurements is about 45 percent of the mean phenotypic value, which provides ample variation for a linkage disequilibrium analysis.

\section{Analysis of variance}

The hypothesis that neocortical volume is in part genomically determined was assessed by analysis of variance (ANOVA), with inbred strain (both parental and recombinant) as the independent variable and residual neocortical volume as the dependent variable, as shown in Table 2 . The main effect of strain was highly significant $\left(F_{35,119}\right.$ $=5.909, p<.0001)$. Significant strain differences were also observed for residual noncortical brain volume $\left(F_{35,119}=\right.$ $8.726, p<.0001)$.
Relative independence of cortical volume and body weight Brain size is known to vary with body size in a wide variety of species, including mice and other rodents $[3,15]$. However, an examination of the regression analyses reported above for phenotype versus predictor variables suggests that the covariation between brain volume and body size is very much weaker for cerebral cortex than it is for noncortical brain volume. We tested this relationship by first computing the Pearson product moment correlation coefficients for body weight and in vivo cortical volume $(r=$ $.224)$ and in vivo noncortical volume $(r=.537)$. We then used Fisher's $r$-to- $z$ transformation [[16], page 49] to statistically test for a significant difference between these correlations. The result provides evidence that the mammalian cerebral cortex is significantly less regulated by factors related to body size than are most other regions of the central nervous system $(z=-3.235, p<0.01)$.

\section{High heritability of brain size}

The proportion of phenotypic variance that might be attributed to genomic factors was estimated as broadsense heritability, or the total genetic determination of a phenotypic trait. Broad-sense heritability was estimated from an analysis of variance with strain as the independent variable and quantitative phenotype as the dependent variable, using the following formula:

$$
\omega^{2}=\frac{F-1}{F+\left(d f_{w}+1\right) / d f_{b}}
$$

where $F$ is the ratio of the mean square deviation between strains to the mean square deviation within strains. The value of $\omega^{2}$ varies between 0.0 and 1.0. These analyses showed that a substantial fraction of phenotypic variance is genetically determined. For regressed cortical volume, $\omega^{2}$ was 0.53; for regressed noncortical volume, $\omega^{2}$ was 0.64 .

\section{QTLs for cortical volume}

Given the high heritability of both residual cortical and noncortical brain volume, the genomic question is not if quantitative trait loci exist for these phenotypic anatomical variables (certainly they must exist or heritability could not be large), but rather where on the genome these QTLs are located.

QTL analyses provide the mechanism for locating genomic regions in which allele values are closely associated with a phenotypic trait. The results of the QTL analysis of 3,795 markers across the mouse genome for association with regressed cortical volume are shown in Figure 1. There are two prominent spikes in the LRS profile for residual cortical volume. Table 3 shows the LRS values produced by marker regression. 


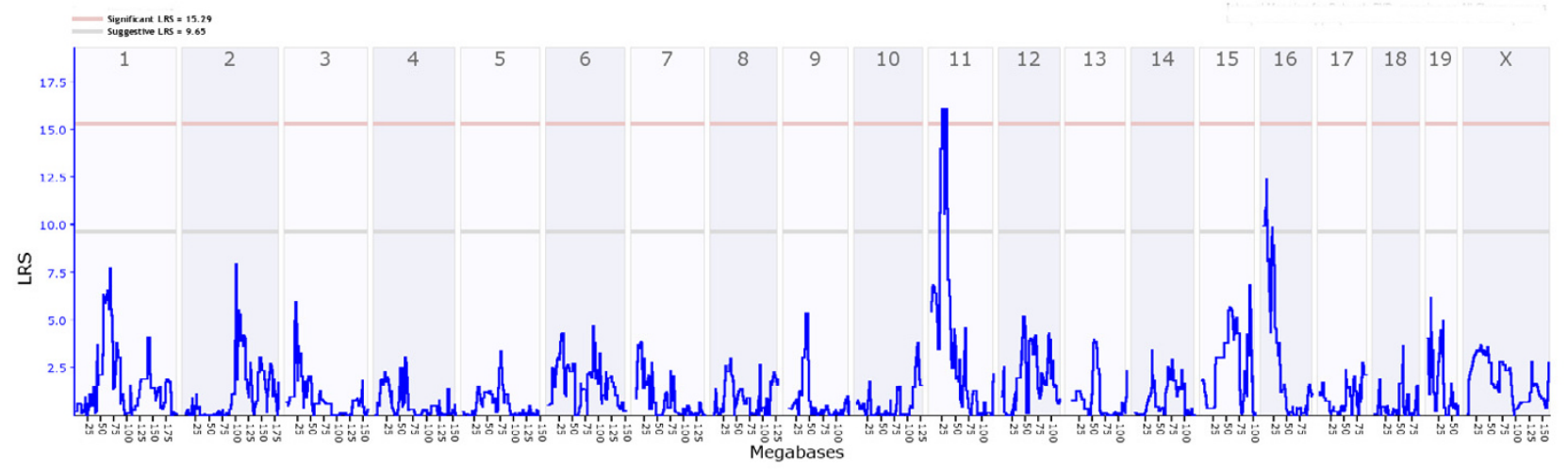

Figure I

QTL Plot for Residual Neocortal Volume. The red upper line indicates the LRS value for genome-wide significant QTL and the lower grey line the level of suggestive QTL according to the criteria of Lander and Kruglyak [33]. There is a genomewide significant linkage disequilibrium located in the proximal portion of chromosome II. There is also a suggestive association on proximal chromosome 16. Notice that there is no significant association between neocortical volume and any marker on chromosome 19.

The most significant marker ( $\mathrm{p} \leq 0.05$ genome-wide) is D11Mit19 at 25.4 megabases (mb) on chromosome 11. Two other nearby markers (S11Gnd020.100 and D11Mit135) also show suggestive association with regressed cortical volume. Because of the restricted genomic resolution afforded by the available number of

Table 3: LRS for regressed cortical and noncortical volume. The criteria LRS values for regressed cortical volume are 21.18 (highly significant at the genome-wide 0.001 level), 17.37 (significant at the genome-wide 0.05 level) and 9.91 (suggestive), following the conventions of Lander and Kruglyak [33]. The corresponding values for regressed noncortical brain volume are 20.90 (highly significant), 17.00 (significant) and 9.89 (suggestive). Genome-wide highly significant findings are marked by double asterisks, significant findings by a single asterisk. Chromosomal position is given in megabases.

\begin{tabular}{llll}
\hline Chromosome & Marker & Position & LRS \\
\hline \multicolumn{4}{c}{ Regressed Cortical Volume } \\
\hline II & DIIMit|9 & 25.4 & $22.66^{* *}$ \\
II & SIIGnf020.100 & 21.0 & $17.45^{*}$ \\
II & DIIMit|35 & 33.7 & 16.39 \\
II & DIIMit23I & 35.8 & 11.45 \\
II & DIIMit5I & 36.7 & 10.14 \\
I6 & DI6Mit 100 & 11.6 & 11.13 \\
I6 & DI6Mit I46 & 23.5 & 10.18 \\
\hline
\end{tabular}

Regressed Noncortical Volume

\begin{tabular}{llll}
\hline 19 & DI9Mit22 & 10.1 & $17.54^{*}$ \\
19 & DI9Mit42 & 0.4 & 13.85 \\
19 & DI9MitI27 & 12.1 & 12.39 \\
11 & DIIMit19 & 25.4 & 12.31 \\
11 & DIIMit23I & 35.8 & 10.38 \\
\hline
\end{tabular}

recombinant inbred strains, we treat these three markers as reflective of a single QTL in the immediate vicinity of D11Mit19.

There is a second, suggestive QTL for residual cortical volume on chromosome 16 . This is reflected by a linkage disequilibrium with two adjacent markers, D16Mit9 (6.0 $\mathrm{mb})$ and D16Mit100 (11.5 mb). These markers on proximal chromosome 16 also contribute to the genetic specification of neocortical volume.

To estimate the minimal proportion of phenotypic variance accounted for by each of these two QTL markers, we calculated the partial eta squared $\left(\eta_{p}^{2}\right)$ from an analysis of variance with the markers D11Mit19 and D16Mit100 and their interaction as factors. These proportions are 0.22 for D11Mit19 and 0.14 for D16Mit100. The proportion attributable to the interaction of the two markers was negligible.

\section{QTLs for noncortical brain volume}

There are significant and suggestive QTLs for regressed noncortical brain volume as well, two of which do not affect cortical volume, and the third affects both, but to differing degrees. Figure 2 presents the corresponding QTL analysis for regressed noncortical brain volume.

A significant association between this regressed noncortical volume and the mammalian genotype may be seen on proximal chromosome 19 . This is most evident at marker D19Mit22 (10.0 mb), which reaches genome-wide significance on many - but not all - permutation tests. Because the results of all permutation tests vary slightly from sam- 


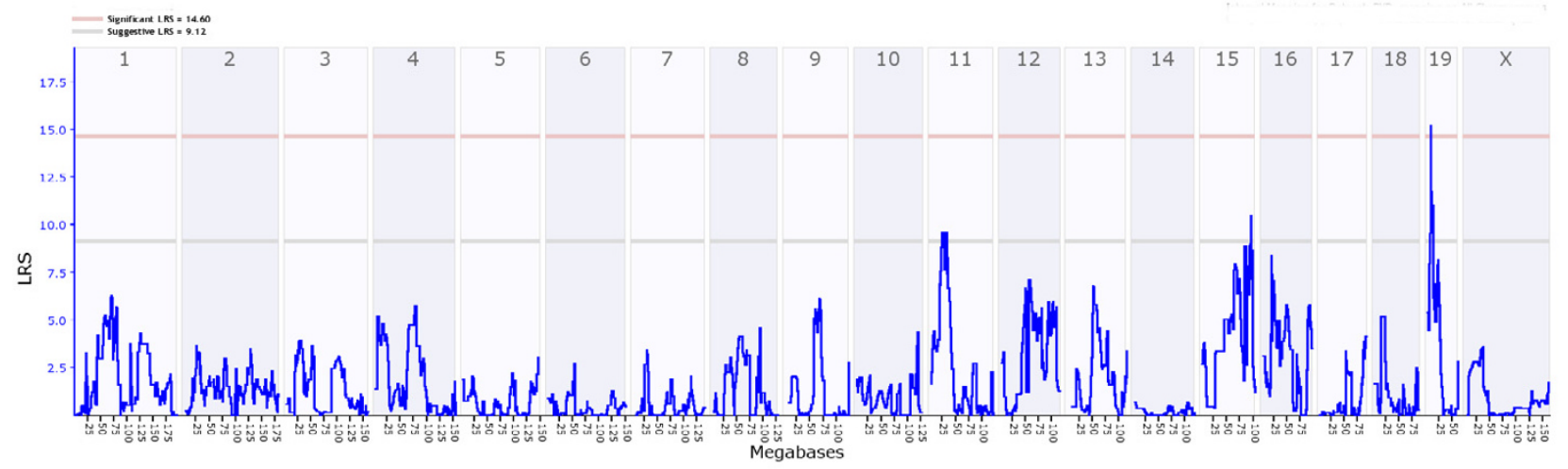

\section{Figure 2}

QTL Plot for Residual Noncortal Volume. Here, the effect of the QTL on proximal chromosome II is attenuated and no longer significant. There is a suggestive association on the distal portion of chromosome I5 and a significant QTL on chromosome 19. Thus, there is a double dissociation between neocortex and noncortex for significant QTLs on chromosomes II and 19.

ple to sample, any LRS score that lies within this narrow range will reach significance with some instances of the permutation test, but not others. Thus, we conclued that this QTL neither greatly exceeds nor falls far short of the genome-wide 0.05 significance level.

This QTL extends to neighboring markers D19Mit42 (9.4 $\mathrm{mb}$ ) and D19Mit127 (12.1 mb). These markers appear to be indicative of a single QTL harboring one or more genes that regulate noncortical, but not cortical, brain size.

D11Mit19 on Chromosome 11, which is significantly related to regressed cortical volume, also shows a suggestive, but not significant, association with noncortical brain volume.

There is also a marker on chromosome 15 with a borderline LRS in marker regression, D15MIT72 $(84.5 \mathrm{mb})$, which exceeds the criterion for a suggestive LRS on some permutation tests, but not on others.

The minimal proportion of phenotypic variance accounted for by these three markers was estimated by $\eta_{p}^{2}$. The obtained values were 0.25 for D19Mit22, 0.13 for D11Mit19, and 0.13 for D15Mit72. All interactions produced negligible values of $\eta_{p}^{2}$.

\section{Composite interval mapping}

Composite interval mapping [17] can increase the sensitivity of a search for additional QTLs associated with a phenotype by incorporating the variance associated with previously identified significant QTLs to the background of the additive model. Composite interval maps were con- structed for both residual phenotypes, but no additional QTLs emerged from these analyses.

\section{Epistasis}

Epistasis is the interaction of two or more genes at different chromosomal sites, which can either be suppressive or facilitative. It is important to test for epistasis, as it indicates a departure from additivity in polygene action.

We tested for epistasis between all the possible marker pairs for the regressed neocortical and noncortical volume phenotypes, respectively, using Ljungberg's DIRECT global optimization algorithm as implemented in WebQTL with phenotype data from the recombinant inbred strains [18]. There were no significant interactions for either phenotypic variable.

\section{Discussion}

These results have several implications for understanding the genetic specification of neocortical development.

\section{Relative independence of cortical size from body size}

The various strains of BXD mice differ considerably from each other in both cortical and noncortical brain volume. However, these two compartments of the mouse brain show quite different dependencies on body size. Nearly 30 percent of the variance in noncortical brain volume can be accounted for by body size in our data (see Striedter [19] for a history of the study of brain and body size).

In contrast, our measurements suggest that neocortical brain volume is much less dependent on body size, with only about 5 percent of that compartment being predictable from body weight. Neocortex appears to be regulated 
by factors that differ substantially from those regulating other regions of the central nervous system, a conclusion that complements recent observations made by Bush and Allman [4].

Thus, one might expect different genomic regions to regulate the heritable factors that determine size of the neocortex and noncortical brain regions, which proves to be the case.

\section{A cortex-dependent QTL on chromosome II}

One QTL with genome-wide significance for regressed cortical volume emerged from our analyses. It was located at the DNA marker D11Mit19 and flanked by two suggestive markers.

A QTL associated near this location (marker D11Mit53 at 16.0 centiMorgans) was reported previously in a doctoral dissertation in which the phenotype was total brain weight, controlling for the effects of sex, age, body weight, litter size, and parity by multiple regression [20]. The QTL was interpreted as one that controls neuron number throughout the brain.

Our data suggest a more specific interpretation. Using our phenotypic data we can compare the the proportion of variance accounted for $R^{2}$ on chromosome 11 at D11Mit19 for neocortex alone (0.34), whole brain volume or brain weight $(0.29)$, and noncortex $(0.21)$, all regressed for body weight and the logarithm of age. The $R^{2}$ values are highest for neocortex, lowest for noncortex, and intermediate for the whole brain. It is clear that this QTL is not a generic determinant of brain size, but rather selectively exerts its effects on the neocortex and perhaps some adjacent forebrain structures such as the olfactory bulb.

\section{Candidate genes for a cerebral cortex volume modulator} The QTL analyses presented above describe chromosomal regions in which a phenotypic measurement - here cortical or noncortical brain volume - are statistically linked with chromosomal markers. But the true endgame of neurogenomics is the discovery of specific genes that mediate these associations. We describe three approaches for narrowing this search.

The mouse chromosome 11 is slightly less than 123 megabases $(\mathrm{mb})$ in length. The strongest linkage between cortical volume and any marker in the murine genome is at D11Mit19 at $25.5 \mathrm{mbp}$ on chromosome 11 . The $\pm 95 \%$ confidence intervals for this QTL are between 20 and 35 $\mathrm{mb}$.

Using the criteria described above, SNP filtering revealed polymorphisms in the coding or untranslated regulatory regions of twenty known genes. The filtered known genes within the chromosome 11 confidence interval for neocortex are E030025D05Rik, AW011752, 4931428D14Rik, C330012F17Rik, 2010316F05Rik, 2510006C20Rik, Stc2, Il9r, 3300001G02Rik, Rhbdf1, Mpg, Hba-x, A230090E05, Stk10, Fbxw1b, Fgf18, Gabrp, Kcnmb1, Dock2, and Vrk2. However, a detailed examination of these genes suggests that they are not uniquely expressed in brain and thus are less likely to provide an explanation of the neocortex QTL.

Nonetheless, several possibilities remain. First, and most obvious, is that the gene(s) controlling neocortical volume have simply not yet been discovered, a possibility that can not be excluded, particularly since the study of the genome is still young.

Another possibility is that the gene may have been "discovered" but its effects on the developing forebrain are not yet known. In this case, the known gene would not be selected in the literature search.

A third possibility is that the gene is known, but was excluded in SNP filtering. Thus, it is of interest that there is a known gene within the QTL on chromosome 11 that is specifically related to neocortex development. Otx1 (chromosome 11 at $21.8 \mathrm{mb}$ ) plays a major and selective role in the development of the mammalian neocortex $[21,22]$. Otx1 is expressed early in development and provides a molecular basis for the development of the deep layers of the cerebral cortex, as well as in the development of the eye, olfactory system, and cochlea.

Otx1 is closely related to Otx2, which modulates the development of the mammalian brainstem. Interestingly, the expression of Otx2 is regulated by two distant enhancers, one located $75 \mathrm{~kb}$ upstream and the other $115 \mathrm{~kb}$ downstream from Otx2 itself on chromosome 14 [23]. If similar enhancers exist for Otx1, they would have been excluded by our SNP filtering procedure. A search of the Celera database reveals that there are $85 \mathrm{D} / \mathrm{B}$ SNPs within \pm 150 $\mathrm{Kb}$ of Otx1. If any of these SNPs lay within a putative Otx1 enhancer, it could provide a molecular basis for the QTL. Thus, Otx1 may still be a reasonable candidate gene mediating the effects of the chromosome 11 QTL for neocortical volume.

\section{A noncortex-dependent QTL on chromosome 19}

In the analysis of regressed noncortical volume, the single QTL detected with genome-wide significance was on proximal chromosome 19. This genomic region had absolutely no effect on regressed cortical volume. Thus, the data display a classical pattern of double dissociation: one factor that affects cortical volume more than noncortical volume and a second factor that shows the mirror image pattern. Double dissociation is an important criterion in 
neuropsychology, because it provides positive logical proof of the specificity of effects. The same argument can be made in the present case as well.

\section{Candidate genes for a noncortical volume modulator}

In an analysis similar to that performed for neocortex we carried out a SNP filtered Celera search of chromosome 19 , which is about $60 \mathrm{mb}$ in length, in the vicinity of D19Mit22, using a QTL confidence interval 13 to $40 \mathrm{mb}$. This search produced 23 known genes with SNPs differentiating the two parental strains. The filtered known genes within the chromosome 19 confidence interval for noncortex are 5730596K20Rik, D630002G06, Slc22a6, Chrm1, Slc3a2, 4930563M09Rik, Gng3, B3Gat3, 0710001O03Rik, BC021917, 1110006I15Rik, Zp1, MOR239-5, A33000E03Rik, MOR202-8, Keg1, Cntf, MOR202-3, MOR202-15, MOR202-36, MOR266-8, MOR2127-1, and MOR212-2.

Examination of expression information for these 23 known genes in the NCBI Entrez Gene database revealed one gene that was particularly promising as a candidate to mediate the QTL's effect on noncortical brain volume. That gene is ciliary neurotrophic factor (Ctnf).

Ctnf is a one of the gp130 family of cytokines and acts as a survival factor for a number of different neuronal cell types. Originally discovered as a factor that supports the survival of parasympathetic nerve fibers in the ciliary ganglia, Ctnf is now known to play an active role in neuronal maintenance within the central nervous system [24]. Recent reports have also shown that Ctnf modulates central nervous system neurogenesis as well [25]. Thus, it is not unreasonable to conclude the genetically controlled natural variations in Ctnf expression might modulate brain size in BXD recombinant inbred mice. Ctnf is indeed a strong candidate gene for understanding the QTL on chromosome 19 related to noncortical brain volume.

\section{Other suggestive QTLs}

The other suggestive QTLs reported above should be noted, although the evidence for them is not exceptionally strong. These include possible cortex-dependent QTL on proximal 16 and the noncortex dependent QTL on chromosome 15 . Suggestive QTLs in today's analyses can provide guidance and support for other, more sensitive, analyses in the future if they are replicated.

\section{Conclusion}

Our localization of QTLs regulating neocortical size may have broader, more cognitive implications. Gibson, in a recent theoretical paper on brain size and mental capacity [[2], p.17], concludes: perhaps "those genetic factors that control enhanced brain size, especially the size of the sensori-motor control for the oral and manual organs, the neocortical association areas, the basal ganglia, and the cerebellum would emerge as the prime genetic determinants of the mental abilities of human infants." Properly designed QTL analyses using the appropriate behavioral phenotypic measures in recombinant inbred mice could help resolve this question.

Since there is ample conservation in the mammalian genome over species, one hopes that findings obtained using well-developed recombinant inbred strains of mice will be applicable to understanding primate and human neurogenomics in a straightforward manner as well.

\section{Methods}

Mice and specimen preparation

All specimens and associated demographic data were obtained from the Mouse Brain Library or MBL [26,27]. (See http://www.mbl.org/tutorials/MBLTrainingManual/ index.html for details.)

All brains were prepared using standard procedures [28]. Each brain was embedded in celloidin and sectioned in 30 micron sections in the coronal plane. Individual sections were stained with cresylechtviolett. Every tenth section was used for measurement, giving a measurement slice thickness of $0.3 \mathrm{~mm}$.

\section{Histological phenotypes}

Histological phenotypes were computed from a set of .3 $\mathrm{mm}$ coronal sections by stereological analysis. We defined two raw histological phenotypes, the in vivo cortical volume and in vivo noncortical volume. Demographic data including fresh brain weight, total body weight, age, and sex were obtained for each mouse from the MBL [29].

\section{In vivo cortical brain volume}

In vivo cortical volume was determined for each mouse using the NIH ImageJ digital measurement system. First, the area of the neocortex as it appears in the serial sections was measured by manually tracing the neocortex. Since the boundary of the molecular layer was extremely variable in some images, the dorsal boundary of the neocortex was taken as the inner edge of the molecular layer, which was clearly discernable in all images. The resulting measurements were then corrected for molecular layer area by a factor determined from images in which both the inner and outer boundaries were clear. In the posterior coronal sections, the lateral boundary of the neocortex was taken as the neocortical-piriform cortex border.

Shrinkage during tissue processing is significant and variable, but can be compensated for to produce accurate estimates of in vivo cortical volume. To do so requires measuring the total volume of the processed specimens, which was calculated using ImageJ. The contrast and 
Table I: Summary of neocortical and related phenotypic measures in 34 BXD recombinant inbred strains and their parental strains. Measured neocortical volume was corrected for the effects of shrinking produced by fixation and embedding in celloidin by multiplying each value by the ratio of the original brain weight to the measured fixed total brain volume. Since the specific gravity of fresh brain is just slightly more than 1.00 , brain weight in grams can be taken as a close approximation of brain volume in cubic centimeters. Thus, in vivo brain volume was estimated by fresh brain weight in grams, as provided for each mouse by the Mouse Brain Library. Noncortical volume was estimated as measured total volume minus neocortical volume and was similarly corrected for shrinkage.

\begin{tabular}{|c|c|c|c|c|c|}
\hline Strain & $\mathbf{N}$ & In Vivo Cortical Volume & $\begin{array}{l}\text { In Vivo Non-cortical } \\
\text { Volume }\end{array}$ & $\begin{array}{l}\text { Regressed Cortical } \\
\text { Volume }\end{array}$ & $\begin{array}{l}\text { Regressed Non-cortical } \\
\text { Volume }\end{array}$ \\
\hline C57BL/6] & 4 & 115.59 & 362.01 & 123.27 & 296.84 \\
\hline $\mathrm{DBA} / 2 \mathrm{~J}$ & 4 & 91.92 & 293.81 & 104.63 & 245.17 \\
\hline BXDI & 5 & 118.47 & 337.57 & 126.78 & 291.27 \\
\hline $\mathrm{BXD2}$ & 5 & 99.58 & 340.76 & 106.36 & 274.38 \\
\hline BXD5 & 5 & 125.11 & 391.73 & 136.07 & 343.66 \\
\hline BXD6 & 4 & 90.41 & 289.17 & 103.1 & 253 \\
\hline BXD8 & 5 & 101.54 & 313.72 & 111.48 & 260.54 \\
\hline BXD9 & 3 & 107.47 & 324.06 & 117.53 & 278.23 \\
\hline BXDII & 3 & 108.96 & 311.68 & 121.34 & 277.88 \\
\hline BXDI2 & 5 & 105.06 & 338.96 & 114.15 & 281.95 \\
\hline BXDI3 & 5 & 103.77 & 316.63 & 110.28 & 259.95 \\
\hline BXDI4 & 5 & 103.47 & 340.79 & 111.53 & 276.46 \\
\hline BXDI5 & 3 & 119.99 & 331.91 & 128.03 & 261.79 \\
\hline BXDI6 & 5 & 115.06 & 343.66 & 121.32 & 285.37 \\
\hline BXDI8 & 4 & 103.33 & 317.7 & 112.84 & 261.64 \\
\hline BXDI9 & 4 & 90.66 & 324.69 & 101.64 & 279.25 \\
\hline BXD20 & 4 & 97.1 & 300.73 & 107.85 & 254.04 \\
\hline BXD2I & 4 & 111.76 & 320.44 & 122.58 & 273.95 \\
\hline BXD22 & 5 & 108.19 & 342.91 & 116.05 & 283.27 \\
\hline BXD23 & 5 & 100.65 & 328.09 & 112.47 & 283.28 \\
\hline BXD24 & 4 & 99.81 & 310.74 & 108.36 & 254.49 \\
\hline BXD25 & 5 & 99.65 & 293.39 & 113.57 & 264.54 \\
\hline BXD27 & 4 & 93.83 & 266.62 & 105.01 & 214.3 \\
\hline BXD28 & 4 & 89 & 304.6 & 97.9 & 255.09 \\
\hline BXD29 & 4 & 84.53 & 295.47 & 96.48 & 252.27 \\
\hline BXD30 & 4 & 77.29 & 269.43 & 90.87 & 237.01 \\
\hline BXD3I & 3 & 95.4 & 312.87 & 103.1 & 252.95 \\
\hline BXD32 & 3 & 99.07 & 357.4 & 106.77 & 289.71 \\
\hline BXD33 & 5 & 102.47 & 317.51 & 110.98 & 273.87 \\
\hline BXD34 & 5 & 101.59 & 309.03 & 106.29 & 249.39 \\
\hline BXD35 & 4 & 105.73 & 313.2 & 114.86 & 268.98 \\
\hline BXD36 & 5 & 102.7 & 301.32 & 113.49 & 261.82 \\
\hline BXD38 & 4 & 98.36 & 307.59 & 108.29 & 266.48 \\
\hline BXD39 & 5 & 101.14 & 307.1 & 108.94 & 261.01 \\
\hline BXD40 & 5 & 100.47 & 325.27 & 111.04 & 286.65 \\
\hline BXD42 & 4 & 101.05 & 352.17 & 108.89 & 303.33 \\
\hline
\end{tabular}

brightness of all digitized sections were adjusted to permit measurement using the ImageJ particle analysis feature. This automatic feature was highly reproducible. To correct each individual mouse brain for shrinkage, the measured specimen neocortical volume was multiplied by the ratio of fresh brain weight to measured total specimen brain volume, assuming a brain density of about $1.05 \mathrm{mg} / \mathrm{mm}^{3}$ in fixed tissue. This restores the volume lost from the fresh brain during histology. The average volumetric shrinkage was $64 \%$, which corresponds to a linear shrinkage of $29 \%$.
Although there is considerable differential shrinkage between specimens, shrinkage is constant across brain structures within each individual brain [4].

\section{In vivo noncortical brain volume}

Noncortical brain volume was calculated by first subtracting measured specimen cortical volume from the measured specimen total brain volume, and then correcting for shrinkage in the manner described above. Thus, for each mouse, the sum of its in vivo cortical and noncortical brain volumes equals its fresh brain weight. 


\section{Single Nucleotide Polymorphism (SNP) filtering}

We interrogated the Celera Discovery System mouse refSNP database for SNPs within the confidence interval of each QTL following the procedures of Yan (2004). SNPs are single nucleotide polymorphisms, or places on the DNA molecule where different strains of mice exhibit different nucleotides.

Because the BXD mice are recombinant inbred strains arising from $\mathrm{C} 57 \mathrm{BL} / 6 \mathrm{~J}$ and $\mathrm{DBA} / 2 \mathrm{~J}$ mice, we filtered the search to include only SNPs that occur between these two parental strains (B-D SNPs). For further analysis, we selected only B-D SNPs that a] occurred within the coding region of the gene as either a mis-match or nonsense mutation, or b] were within the 5'-regulatory region, or c] were present in the 3'-untranslated region. Silent SNPs and SNPs in the intronic or intergenic regions were excluded.

Finally, only SNPs associated with known genes, as determined by a search of the NCBI Entrez Gene database [31], were included. It this way, we were able to identify all known genes within the confidence interval of each QTL.

We also searched for evidence that each of these known genes is expressed morphologically within the brain using Mouse Genome Informatics databases at the Jackson Laboratory [32].

Analyses of these data address a number of issues regarding the genomic determination of neocortical development. These include the natural variation of neocortical and noncortical size in relation to body size, their heritabilities, the localization of the most important genomic regions responsible for both quantities, and identification of known genes that could mediate the observed QTLs.

\section{Authors' contributions}

The intellectual contributions to this paper were shared evenly between the two authors. RL bore primary responsibility for stereological measurement and JB was the principal author of the manuscript.

\section{Acknowledgements}

This project is supported by the Human Brain Project under National Institute of Mental Health grant I K07MHO I 953, National Institute on Deafness and Other Communication Disorders DC/8424559, and the National Science Foundation MH/8429337. We thank Nan Cheng for help in stereological measurement, and Gerhard Hellemann for statistical discussions.

\section{References}

I. Blake DT, Byl NN, Merzenich MM: Representation of the hand in the cerebral cortex. Behav Brain Res 2002, I35(I-2): I79-184.

2. Gibson KR: Evolution of human intelligence: The roles of brain size and mental construction. Brain, Behavior, and Evolution 2002, 59:10-20.

3. Finlay BL, Darlington RB, Nicastro N: Developmental structure in brain evolution. Behavioral and Brain Sciences 200I, 24:263-308.
4. Bush EC, Allman JM: The scaling of frontal cortex in primates and carnivores. Proceedings of the National Academy of Sciences of the United States 2004, I 0 I:3962-3966.

5. Blinkov SM, Glezer II: The Human Brain in Figures and Tables New York: Basic Books; 1968.

6. Baare WFC, Pol HEH, Boomsma DI, Posthuma D, de Geus EJC, Schnack HG, van Haren NEM, van Oel CJ, Kahn RS: Quantitative genetic modeling of variation in human brain morphology. Cerebral Cortex 2001, I I:816-824.

7. Reiss AL, Abrams MT, Singer HS, Ross JL, Denckla MB: Brain development, gender and IQ in children. A volumetric imaging study. Brain 1996, I 19:1763-1774.

8. Thompson PM, Cannon TD, Narr KL, van Erp T, Poutanen VP, Huttunen M, Lonqvist J, Standerskjold-Nordenstam CG, Kaprio J, Khaledy $\mathrm{M}$, Dail R, Zoumalan $\mathrm{Cl}$, Toga AW: Genetic influences on brain structure. Nature Neuroscience 200 I, 4(I 2): I 253-I 258.

9. Posthuma D, DeGeus EJC, Baare WFC, Pol HEH, Kahn RS, Boomsma $\mathrm{DI}$ : The association between brain volume and intelligence is of genetic origin. Nature Neuroscience 2002, 5(2):83-84.

10. Chesler EL, Wang J, Lu L, Qu Y, Manly KF, Williams RW: Genetic correlates of gene expression in recombinant inbred strains: A relation model system to explore neurobehavioral phenotypes. Neuroinformatics 2003, 1:343-358.

II. Wang J, Williams RW, Manly KF: Web-based complex trait analysis. Neuroinformatics 2003, I:229-308.

12. Doerge RW, Churchill GA: Permutation tests for multiple loci affecting a quantitative character. Genetics 1996, 142:285-294.

13. Van Ooijen JW: Accuracy of mapping quantitative trait loci in autogamous species. Theoretical and Applied Genetics 1992, 84:803-8I I.

14. Kim JJ, Davis SK, Taylor JF: Application of non-parametric bootstrap methods to estimate confidence intervals for QTL location in a beef cattle QTL experimental population. Genetical Research 2002, 79:259-263.

15. Jerison HJ: Evolution of the Brain and Intelligence New York: Academic Press; 1973.

16. Cohen J, Cohen P, West SG, Aiken LS: Applied multiple Regression/Correlation Analysis for the Behavioral Sciences 3rd edition. Mahwah, N.J.: Lawrence Erlbaum Associates; 2003.

17. Jansen RC: Interval mapping of multiple quantitative trait loci. Genetics 1993, I 35:205-2II.

18. Ljungberg K, Holmgren S, Carlborg O: Simultaneous search for multiple QTL using the global optimization algorithm DIRECT. Bioinformatics 2004: I887-1895.

19. Striedter GF: Principles of Brain Evolution Sunderland, Md.: Sinauer Associates, Inc; 2005.

20. Strom RC: Genetic Analysis of Variation in Neuron Number. In PhD thesis University of Tennessee, Memphis; 1999.

21. Acampora D, Gulisano M, Broccoli V, Simeone A: Otx genes in brain morphogenesis. Progress in Neurobiology 200I, 64:69-95.

22. Panto MR, Zappala A, Tuorto F, Cicirata F: Role of the Otx I gene in cell differentiation of mammalian cortex. European Journal of Neuroscience 2004, 19:2893-2902.

23. Kurakawa D, Kiyonari H, Nakayama R, Kimura-Yoshida C, Matsou I, Aizawa S: Regulation of Otx2 gene expression and its functions in mouse forebrain and midbrain. Development 2004, I3 I (14):3319-3331.

24. Lo DC: A central role for ciliary neurotrophic factor? Proceedings of the National Academy of Sciences USA 1993, 90:2557-2558.

25. Emsley JG, Hagg T: Endogenous and exogenous ciliary neurotrophicc factor enhances forebrain neurogenesis in adult mice. Experimental Neurology 2003, I 83:298-310.

26. Rosen GD, Williams AG, Capra JA, Connolly MT, Cruz B, Lu L, Airey DC, Kulkarni K, Williams RW: The Mouse Brain Library @ http:I Iwww.mbl.org. International Mouse Genome Conference 2000.

27. Williams RW: Mapping genes that modulate mouse brain development: A quantitative genetic approach. In Mouse Brain Development Edited by: Goffinet AF, Rakic P. New York: Springer-Verlag; 2000:21-49.

28. Rosen GD, Williams RW: Complex trait analysis of the mouse striatum: Independent $Q T L s$ modulate volume and neuron number. BMC Neuroscience 2001, 2:.

29. Rosen GD, La Porte NT, Diechtiareff B, Pung CJ, Nissanov J, Gustafson C, Bertrand L, Geven S, Fan Y, Tretiak OJ, Manly KF, Park MR, Williams AG, Connolly MT, Capra JA, Williams RW: Informatics 
center for mouse genomics: The dissection of complex traits in the nervous system. Neuroinformatics 2003, 1:327-342.

30. Yan Y, Wang M, Lemon WJ, You M: Single nucleotine polymorphism (SNP) analysis of mouse quantitative trait loci for identification of candidate genes. Journal of Medical Genetics 2004, 4I: III.

31. Pruitt KD, Magloff DR: RefSeq and LocusLink: NCBI gene-centered resources. Nucleic Acids Research 200I, 29:137-I40.

32. Mouse Genome Database Group: The Mouse Genome Database (MGD): integrating biology with the genome. Nucleic Acids Research 2004, 32:D476-8I.

33. Lander E, Kruglyak L: Genetic dissection of complex trains: Guidelines for interpreting and reporting linkage results. Nature Genetics 1995, I I:24 |-247.

Publish with Bio Med Central and every scientist can read your work free of charge

"BioMed Central will be the most significant development for disseminating the results of biomedical research in our lifetime. "

Sir Paul Nurse, Cancer Research UK

Your research papers will be:

- available free of charge to the entire biomedical community

- peer reviewed and published immediately upon acceptance

- cited in PubMed and archived on PubMed Central

- yours - you keep the copyright

Submit your manuscript here:

http://www.biomedcentral.com/info/publishing_adv.asp
BiolMedcentral 